\title{
Surface Tension Measurements of Liquid Iron-Nickel-Sulphur Ternary System Using the Electromagnetic Oscillating Droplet Technique
}

\author{
Hong-Kee LEE, Martin G. FROHBERG and Jnan P. HAJRA ${ }^{1)}$ \\ Institute of General Metallurgy, Technical University Berlin, Joachimstaler Strasse 31/32, D-1000 Berlin 15, Germany. \\ 1) Formerly Guest Professor, Technical University Berlin. Now at Indian Institute of Science, Bangalore, India.
}

(Received on January 13, 1993; accepted in final form on May 21, 1993)

\begin{abstract}
Surface tensions of liquid iron-nickel-sulphur ternary alloys as a function of the composition and the temperature have been determined by the oscillating droplet method combined with electromagnetic levitation technique. The natural frequency of the oscillating droplet was evaluated using a Fourier analyzer, and the influence of magnetic field strength on the surface tension was also considered. The temperature dependence of surface tension for the liquid iron and iron-nickel melts containing the surface active element sulphur were found to be positive. This effect may be due to the change of the sign in the entropy terms for the bulk and surface phase caused by the reduced mobility of the surface atoms. Furthermore, iso-surface tension diagram for the iron-nickel-sulphur ternary system at $1873 \mathrm{~K}$ is given and discussed.
\end{abstract}

KEY WORDS: electromagnetic oscillating droplet technique; surface tension of iron-sulphur alloys; surface tension of iron-nickel-sulphur alloys; surface active element; positive temperatur coefficient of surface tension; iso-surface tension diagram.

\section{Introduction}

Surface phenomena involving surface active elements play an important role in metallurgical and technical processes. The presence of surface active components, particularly such as oxygen and sulphur, which segregate strongly at the interface, alters substantially the surface tension of the solvent even at very low concentrations. In addition these elements change the temperature dependency of the surface tension for a metallic solution from a negative value to a positive one. From practical point of view, a clarification of such effects may be helpful to understand metallurgical processes in the presence of oxygen and sulphur.

These elements, however, unavoidably can be picked up from the surrounding gas atmosphere or from the refractories during the measurement of surface tension; therefore surface tension values for metallic systems reported in the literature scatter widely.

The contamination problem of specimens could be overcomed by introducing the electromagnetic levitation technique in combination with the oscillating droplet method proposed first by $\mathrm{Lu}$ and co-workers. ${ }^{1-3)}$ The technique permits the containerless melting and heating of electrically conductive materials. The principle of this surface tension measurement technique is based on the relationship between the surface tension and the oscillation frequency of a free falling droplet. ${ }^{4}$ Because of many advantages and its sensibility this experimental technique has intensively been studied and employed in the recent years ${ }^{5-22)}$ measuring the surface tension of molten metals and alloys.

Furthermore, the thermodynamic properties of the undercooled metallic systems are actually investigated to clarify the glass transformation and their homogeneous nucleation. The knowledge of the surface tension of supercooled liquid metals may be also important to study these characteristics along with that of solid state, which may allow to explain the undercooled phenomena of metallic solutions in conjunction with their surface (or interfacial) properties. Surface tensions in undercooled region can problemlessly be determined using the here applied experimental technique by avoiding any heterogeneities.

This experimental technique was adopted in our laboratory ${ }^{23)}$ and the results for some systems were recently published. ${ }^{24,25)}$ In the present work the influence of sulphur on the surface tension of liquid iron and iron-nickel alloys has been investigated.

\section{Experimental}

\subsection{Theoretical Background}

The theoretical treatment of the relationship between the surface tension and the oscillation frequency of a free-falling (or levitated) liquid drop has been derived by Lord Rayleigh ${ }^{4}$ equating the change of the surface energy with the kinetic energy of motion in consequence 
of the surface oscillation. Rayleigh's equation applied for the levitating droplet in an electromagnetic field can be expressed as:

$$
\sigma=\frac{3}{8} \pi M v_{l=2}^{2}
$$

where $\sigma$ is the surface tension of solution, $M$ the mass of a droplet and $v$ the oscillation frequency of the fundamental mode $l=2$.

Under real experimental condition, however, the combined effect of the compensating electromagnetic field and the gravitational force lead to an aspherical equilibrium shape of the oscillating droplet. According to this fact, experimental investigations of the surface tension using the oscillating droplet technique in an electromagnetic field show that the expected single Rayleigh frequency (of the fundamental mode $l=2$ ) is split into either three or five unequally spaced peaks caused by an asphericity of the equilibrium droplet shape. Therefore, simple Rayleigh's equation based on the perfect spherical shape of the equilibrium droplet has to be modified.

Theoretical work to solve this probelm bas been carried out recently by Cummings and Blackburn. ${ }^{26)}$
They have derived the following frequency sum rule to obtain the Rayleigh frequency for split modes:

$$
v_{l=2}^{2}=\frac{1}{5} \sum_{m=-l}^{m=l} v_{l=2, m}^{2}-2 \bar{v}_{t}^{2}
$$

$m$ is the split mode assigned to $l$ and $\bar{v}_{t}^{2}$ is the mean square translational frequency which takes into account the effect of the electromagnetic field strength on the surface tension of the droplet.

A rotating and/or not axisymmetric droplet will show five unequally spaced bands in the spectrum, designated by $l=2, m=-l, \ldots,+l$. In case of a non rotating axisymmetric droplet only three peaks may be expected because two pairs of the five peaks superpose. Equation (2) can for this case be rewritten as:

$$
v_{l=2}^{2}=\frac{1}{5}\left(v_{l=2, m=0}^{2}+2 v_{l=2, m= \pm 1}^{2}+2 v_{l=2, m= \pm 2}^{2}\right)-2 \bar{v}_{t}^{2}
$$

Our experimental observations reveal usually three peaks in the Fourier spectrum. In the present work, Eq. (1) combined with Eq. (3) is used to calculate the surface tension from the Fourier frequency spectrum.

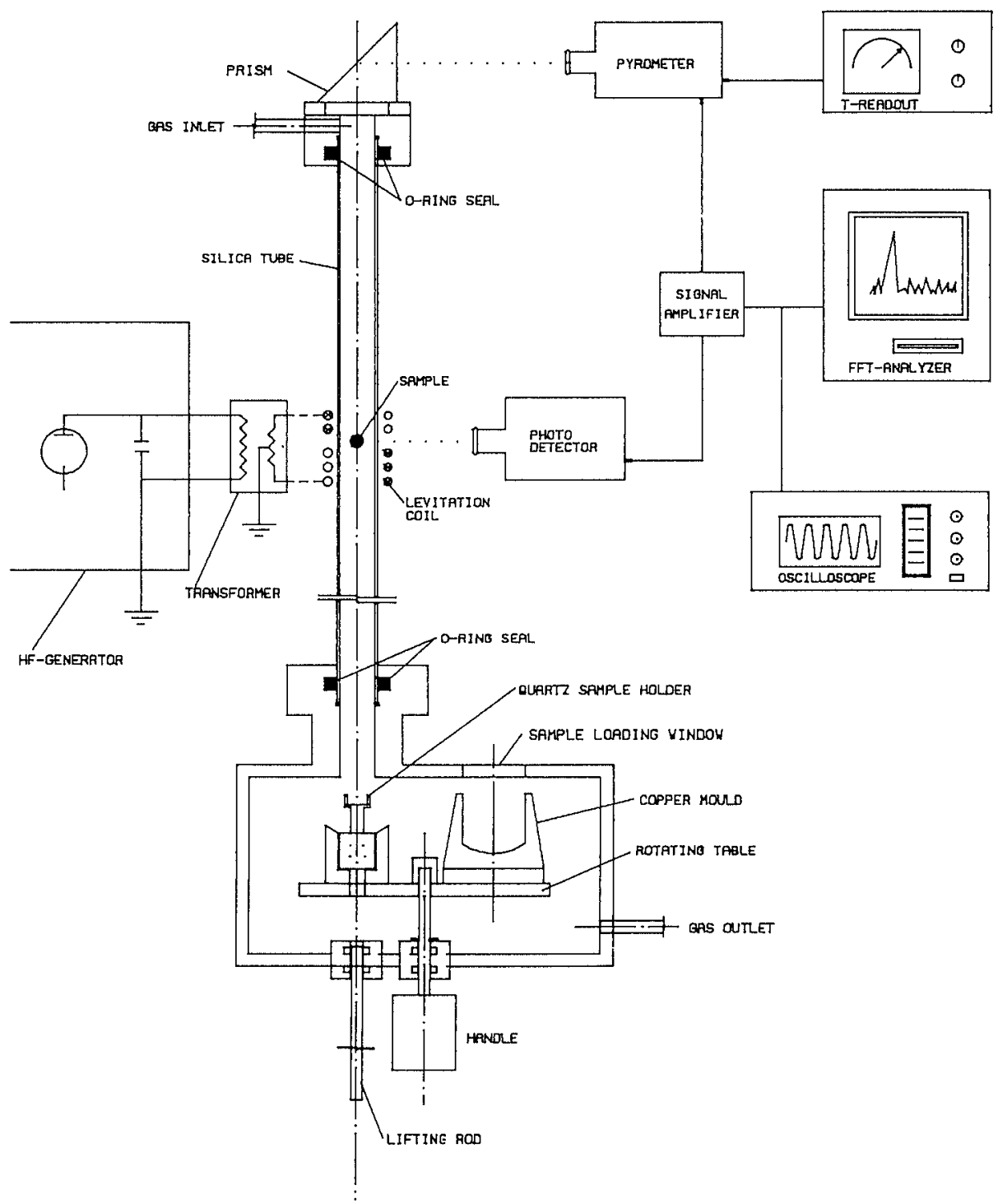

Fig. 1.

Schematic diagram of the experimental arrangement to determine the surface tension of molten metals and alloys by the electromagnetic oscillating droplet technique. 


\subsection{Experimental Arrangement and Procedure}

The experimental apparatus used in the present work is schematically shown in Fig. 1. For the power supply an AEG $25 \mathrm{KW}$ high frequency generator, operating at $400 \mathrm{kHz}$, was used. The silica tube, the outer diameter of which was $16 \mathrm{~mm}$, was placed within a levitation coil and connected to a small vacuum chamber. In this chamber six quartz glass sample holders and six copper quenching moulds were located on a small rotating table. With this arrangement, it was possible to measure six different samples in an experimental unit. The chamber was capable of being evacuated to $10^{-3} \mathrm{~Pa}$.

Temperature regulation of the sample was achieved by varing the composition of the gas mixture and its flow rate. The gases used were highly pure argon and helium $(99.9999 \%)$ and further prepurifed by passing them through a deoxy-cartridge tube to remove oxygen and moisture. Besides the surface contamination of sample was eliminated by hydrogen before its melting in the levitation coil. The temperature of the samples was measured by a modified two-color quotient pyrometer which can also be used to detect the oscillation frequency of levitating droplet. The pyrometer was calibrated by measuring the melting point of pure nickel and iron. The uncertainty associated with the temperature measurement was estimated to be $\pm 5 \mathrm{~K}$ in the temperature range considered.

The motion of a levitated sample was observed via $45^{\circ}$ prism placed on the optical quartz plate and additionally from the side direction. For determination of oscillating frequency the measuring spot of the pyrometer was focused onto the edge of an oscillating sample. A photodetector installed in the pyrometer recorded the change of the incoming light intensity. The output signals fed via a signal amplifier were then transmitted to an analog/ digital oscilloscope, where they were monitored, controled and stored. At the same time, a Fourier transform of the output signals was performed by an FFTanalyser to obtain a frequency spectrum. A second photo detector from the side direction is additionally used to identify the uncertain oscillation or translation frequencies. The experimental error due to the uncertainty in the assignment of the peaks from the frequency spectrum was of the order of $\pm 30 \mathrm{mN} / \mathrm{m}$.

After measuring the oscillation frequency, each sample was quickly quenched by increasing the flow rate of gas and kept for chemical analysis. The samples (weighing from 0.8 to $1 \mathrm{~g}$ ) were prepared in the following manner: into the hole of an iron cylindrical specimen sulphur powder was filled for alloying. The hole was sealed by a nickel plug. By varying the masses of the three components the wanted concentration of the sample could be obtained. The samples supplied by Johnson Matthey were of high purity (99.99\%) and weighed prior to and after the experiments. The loss of the sample weight due to vaporization was always less than about $0.3 \%$. The oxygen and sulfur contents of the samples were determined by a LECO RO-116 and a LECO CS-244 determinator. The contamination of samples by oxygen was less than $1 \mathrm{ppm}$.

The most important part of the experimental technique was actually the finding of the optimum levitation coils which ensure three clearly spaced peaks in the spectrum. There are some useful equations to calculate the behaviour of the sample in the electromagnetic field, ${ }^{27,28)}$ but in practice the design of the optimal coils is additionally a matter of experience. For convenience the coils successfully used in this work are given in Fig. 2.

\section{Results and Discussion}

In our previous papers surface tension values of boundary systems, namely pure elements $\mathrm{Fe}$ and $\mathrm{Ni}$ and binary systems $\mathrm{Fe}-\mathrm{Ni}$ and $\mathrm{Ni}-\mathrm{S}$ were reported. ${ }^{24,25)}$ In this work the results for the binary $\mathrm{Fe}-\mathrm{S}$ and ternary $\mathrm{Fe}-\mathrm{Ni}-\mathrm{S}$ system will be presented.

\subsection{The Iron-Sulphur Binary System}

Figure 3 shows the temperature dependence of the surface tension of iron-sulphur alloys. As already mentioned, it can be seen that in presence of the surface active element sulphur the temperature coefficient in liquid iron exhibits more or less positive values. Thermodynamically, the temperature coefficient is related to the entropy of the bulk $s^{b}$ and the surface phases $s^{\sigma}$ per unit area. ${ }^{29)}$ This can be expressed as:

$$
\frac{d \sigma}{d T} \approx s^{b}-s^{\sigma}
$$

For a metallic solution free from the surface active elements the temperature coefficient of the surface tension is usually negative in consequence of higher excess surface entropy values than that of bulk phase $s^{\sigma}>s^{b}$. It may be supposed that the surface structure of metallic solutions where surface active species are adsorbed is comparable to a tight two-dimensional compound. Because of this stable surface structure the

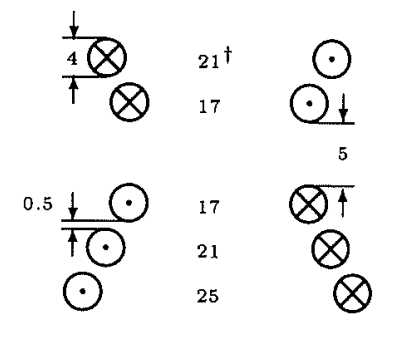

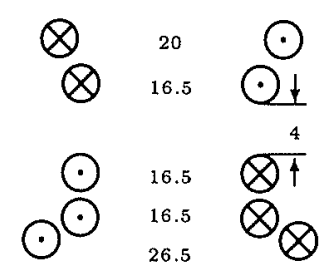

Unit: $m m$

$\dagger$ Inner diameter
Fig. 2.

The levitation coils employed in this work. 


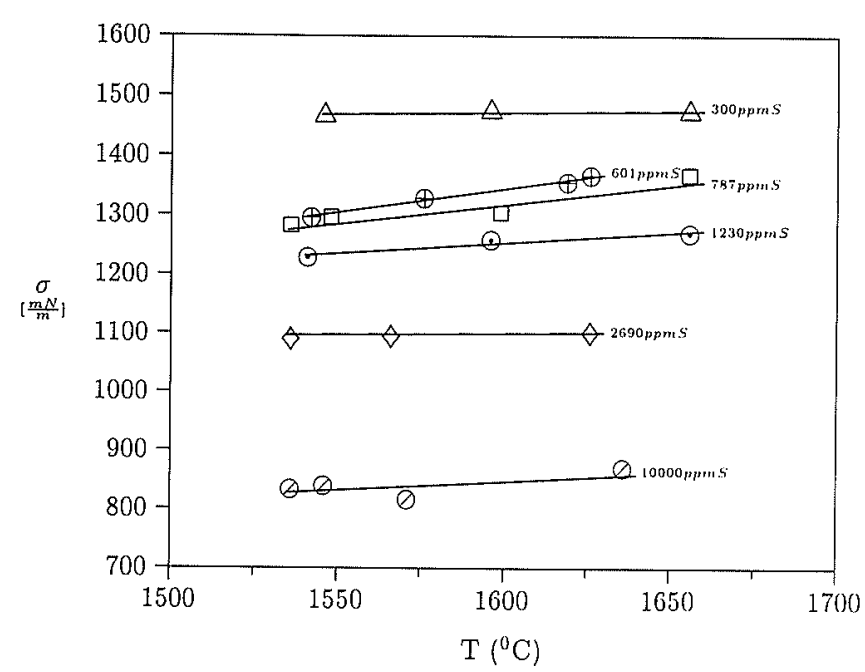

Fig. 3. Variation of the surface tension $\sigma$ of iron-sulphur alloys as a function of the temperature $T$.

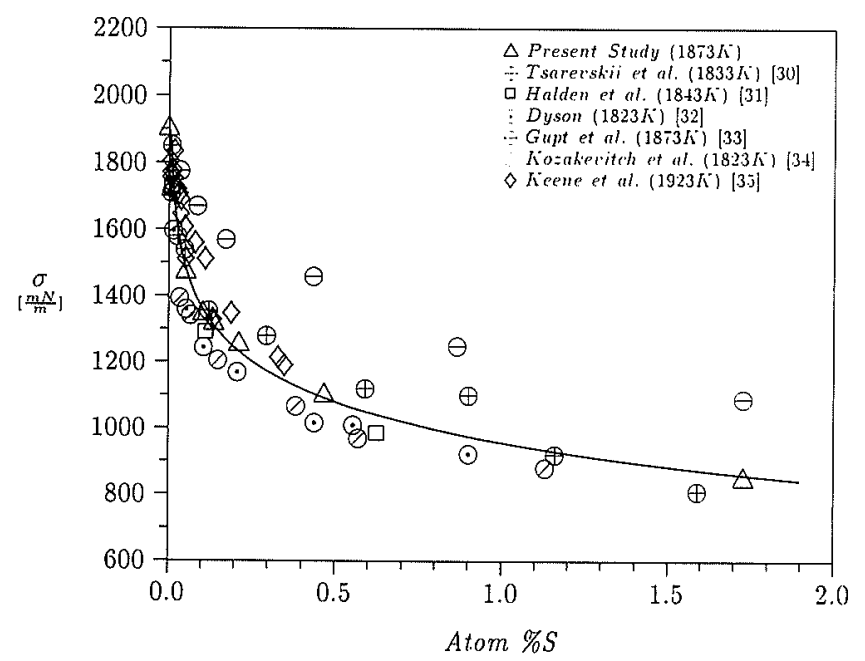

Fig. 4. Variation of the surface tension $\sigma$ with the sulphur content in system iron-sulphur at $1873 \mathrm{~K}$.

mobility of the surface atoms is reduced; this leads to a consequent decrease of the excess surface entropy related to that of bulk phase. The metallic solution containing the surface active elements, therefore, take positive temperature coefficients according to our observation.

Figure 4 shows the comparison of the surface tension of liquid iron-sulphur alloys achieved by the present work with those of other workers. Except the data of Gupt et al. ${ }^{33)}$ there exists a tolerable agreement among the presented values.

The surface activity of sulphur determined from the solpe of $\sigma$ vs. $x_{\mathrm{S}}$ at infinite dilution (Fig. 4), namely $-\left(d \sigma / d x_{\mathrm{s}}\right)_{x_{\mathrm{s}} \rightarrow 0}$, which provides a criterium of the adsorption behaviour of a surface active species, was found from our results for iron-sulphur melts to be $1.6 \times 10^{3} \mathrm{~N} / \mathrm{m}$ at $1873 \mathrm{~K}$. This result shows a reasonable agreement with the value of $1.8 \times 10^{3} \mathrm{~N} / \mathrm{m}$ at $1823 \mathrm{~K}$ reported by Lupis ${ }^{36)}$ accounting the positive temperature dependence of the surface tension.

The surface active element is characterized not only by a high value of the surface activity but also the rapidly reached saturation stage. The relative adsorption of sulphur in iron $\Gamma_{\mathrm{S}}^{(\mathrm{Fe}) \text { sat }}$ determined from the slope of the

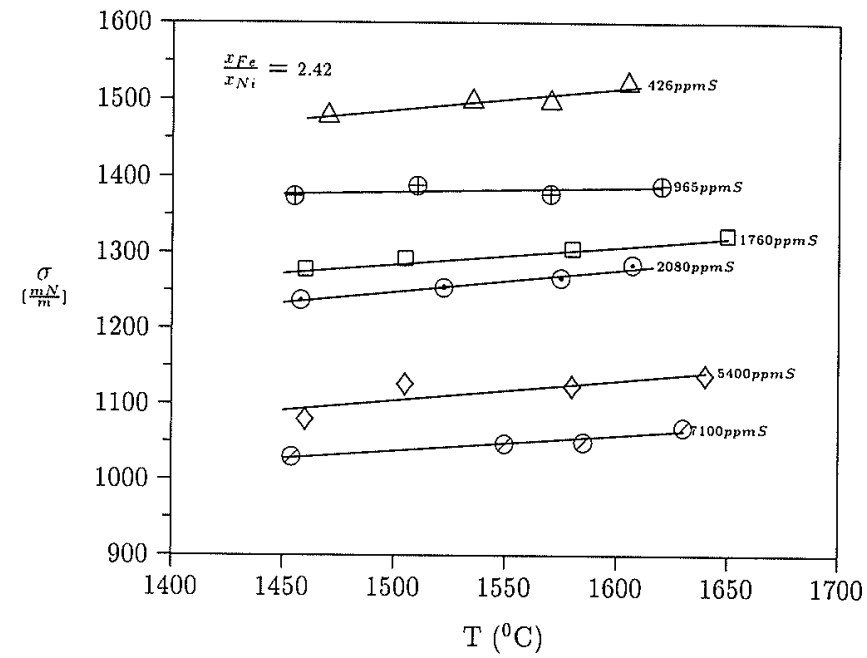

Fig. 5. Variation of the surface tension $\sigma$ of iron-nickelsulphur alloys $\left(x_{\mathrm{Fe}} / x_{\mathrm{Ni}}=2.42\right)$ as a function of the temperature.

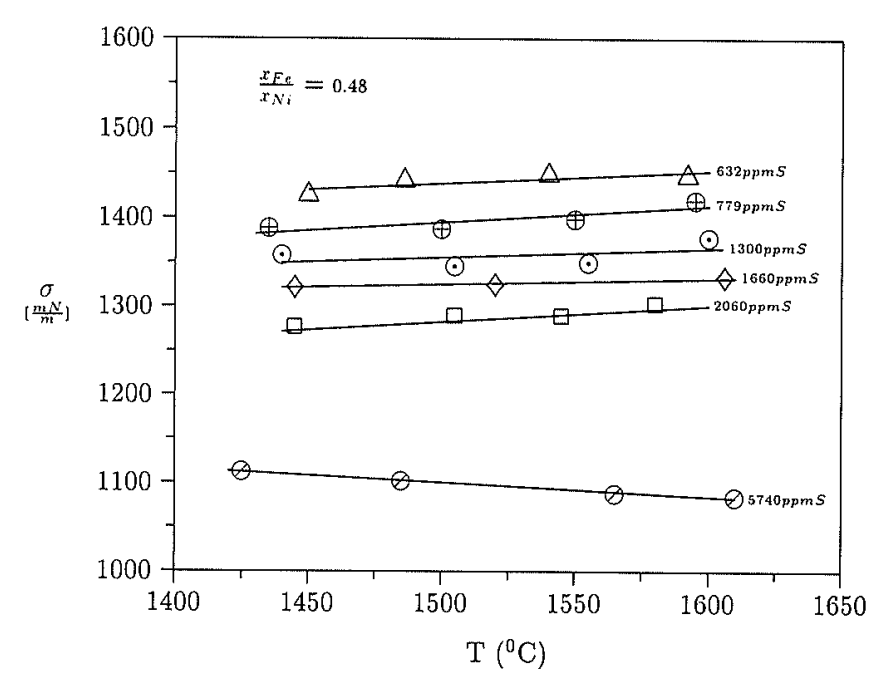

Fig. 6. Variation of the surface tension $\sigma$ of iron-nickelsulphur alloys $\left(x_{\mathrm{Fe}} / x_{\mathrm{Ni}}=0.48\right)$ as a function of the temperature.

plot of $\sigma$ against $\ln x_{\mathrm{S}}$ (Fig. 7) through the Gibbs adsorption isotherm at $1873 \mathrm{~K}$ is given by:

$$
\Gamma_{\mathrm{S}}^{(\mathrm{Fe}) \mathrm{sat}}=-\frac{1}{R T}\left(\frac{\partial \sigma}{\partial \ln x_{\mathrm{S}}}\right)_{x_{\mathrm{Fe}_{\mathrm{e}} \rightarrow 1}}=11.4 \times 10^{-6}\left[\mathrm{~mol} / \mathrm{m}^{2}\right]
$$

Consequently, the area $A_{\mathrm{S}}^{(\mathrm{F}) \text { sat }}$ occupied by the adsorbed sulphur atom at the saturation stage $\left(\Gamma_{\mathrm{S}}^{\text {(Fe)sat }}\right)$ yields a value of about $14.5 \times 10^{-20} \mathrm{~m}^{2} /$ atom. Kozakévitch ${ }^{37)}$ suggested that the surface structure adsorbed by surface active species could be considered as a ionic double layer. The value of the surface area occupied by one sulphur ion proposed by him was $10.7 \times 10^{-20} \mathrm{~m}^{2} /$ atom for a close packed plane and $12 \times 10^{-20} \mathrm{~m}^{2} /$ atom for a (010) plane of $\mathrm{FeS}$, respectively. As in a previous paper $^{25)}$ mentioned, the surface layer may be in equilibrium with surface vacancies formed by the mutual repulsion of metalloid atoms in the adsorbed surface film. Therefore the calculated values of surface area from the experiment should be somewhat greater than those according to the theory of the ionic double layer. 


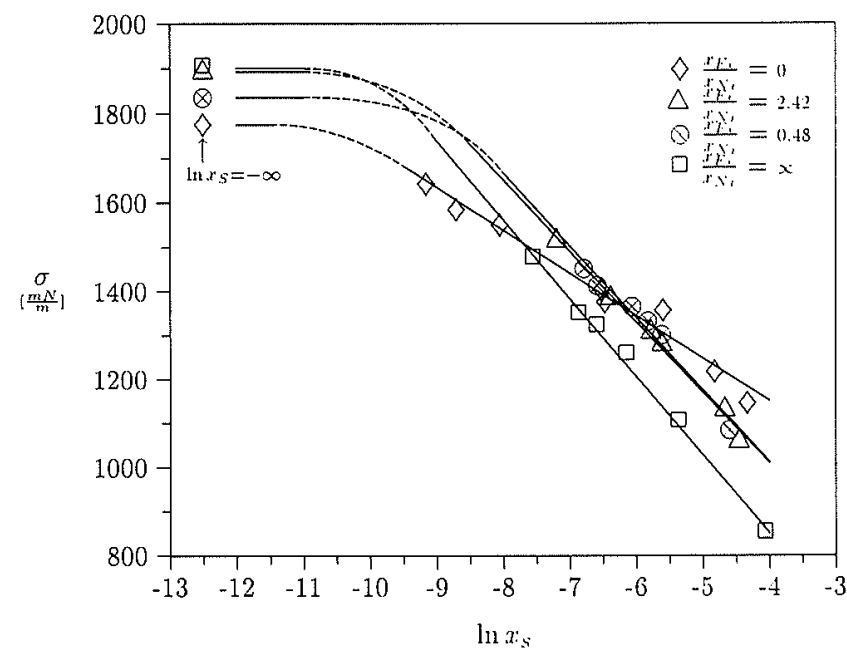

Fig. 7. Surface tension $\sigma$ vs. the logarithm of the atomic fraction sulphur $\ln x_{\mathrm{S}}$ at $1873 \mathrm{~K}$.

\subsection{The Iron-Nickel-Sulphur Ternary System}

The variation of the surface tension for a multicomponent system is mainly dependent of the surface properties (surface areas and surface tensions) of the system-consisting individual pure components along with the thermodynmaic behaviour. Since thermodynamically the system $\mathrm{Fe}-\mathrm{Ni}$ shows a small negative deviation from the ideality together with a slight difference in the surface properties of the pure components iron and nickel, only two compositional paths $x_{\mathrm{Fe}} / x_{\mathrm{Ni}}=$ 2.42 and 0.48 seemed to be necessary to study the surface tension behaviour of the $\mathrm{Fe}-\mathrm{Ni}-\mathrm{S}$ ternary system.

Figures 5 and 6 present the temperature dependences of surface tension for the two compositional paths with different sulphur contents. Beside of one exception $\left(0.574 \mathrm{wt} \% \mathrm{~S}\right.$ for $\left.x_{\mathrm{Fe}} / x_{\mathrm{Ni}}=0.48\right)$ all temperature coefficients are positive. The surface tension of iron-nickelsulphur alloys do not show a strong temperature dependence at all.

Furthermore, the variation of the surface tension with sulphur contents for the compositional paths (Fig. 7) show the same tendency due to the similar physical properties of pure iron and nickel. This result clearly demonstrates the expected predominant role of sulphur in determining the overall surface tension behaviour of the system iron-nickel-sulphur.

The relative adsorption of sulphur in iron-nickel melts for each compositional path $x_{\mathrm{Fc}} / x_{\mathrm{Ni}}=2.42$ and 0.48 was found to be $10.3 \times 10^{-6}$ and $10.5 \times 10^{-6} \mathrm{~mol} / \mathrm{m}^{2}$, respectively. From these data the values for the surface areas occupied by one sulphur atom in iron-nickel melts were calucalted to be $16.1 \times 10^{-20} \mathrm{~m}^{2} /$ atom for $x_{\mathrm{Fe}} / x_{\mathrm{Ni}}=2.42$ and $15.8 \times 10^{-20} \mathrm{~m}^{2} /$ atom for $x_{\mathrm{Fe}} / x_{\mathrm{Ni}}=$ 0.48 . In all cases there are no significant differences in the surface excess properties for these two concentration ratios.

The interpolated iso-surface tension curves based on our data for the $\mathrm{Fe}-\mathrm{Ni}-\mathrm{S}$ system are shown in Figs. 8(a) and 8(b). Because of the small sulphur additions rectangular types of diagrams were preferred. Regarding Fig. 8(a) nearly straight lines of the surface tension between iron and nickel seem to exist up to nearly 0.2
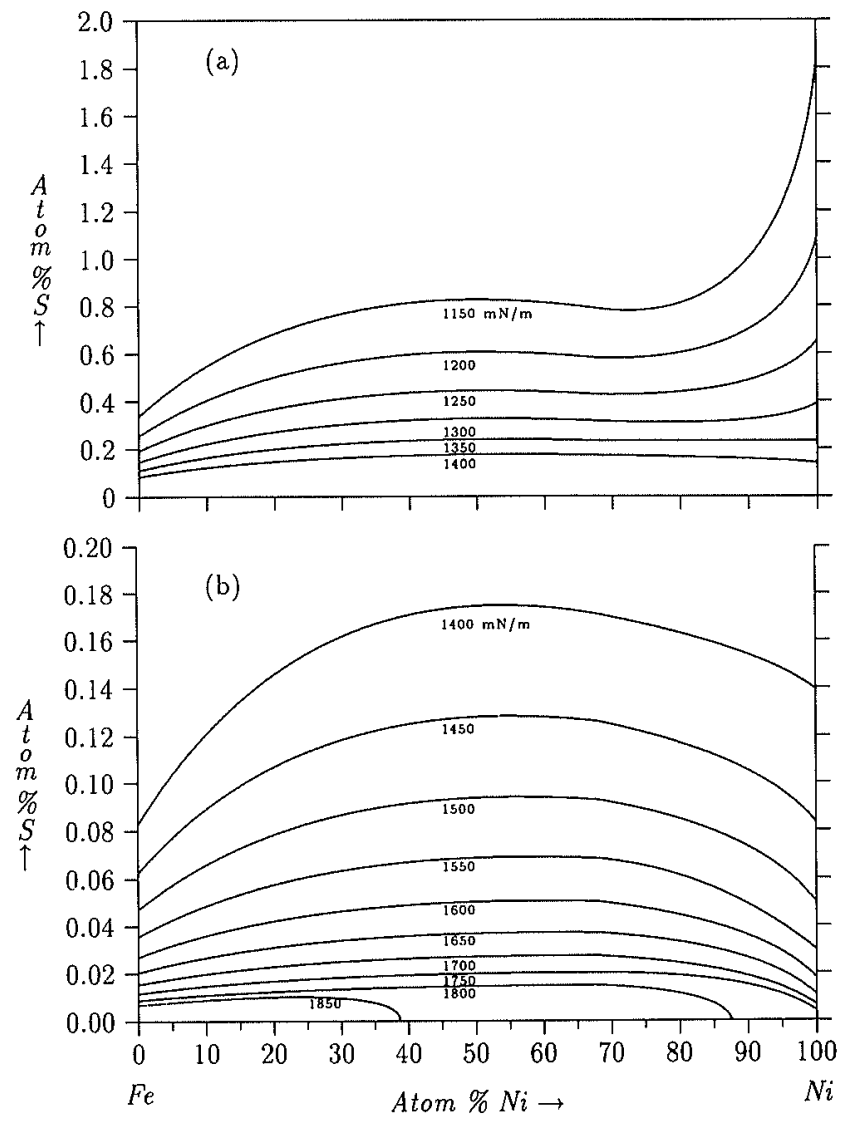

Fig. 8. Iso-surface tension curves for the system iron-nickelsulphur at $1873 \mathrm{~K}$.

at $\%$ sulphur. Enlarging the scale (Fig. 8(b)) the deviation from linearity from 0.02 at $\%$ sulphur is obvious. At sulphur contents $>0.2$ at $\%$ the individual adsorption characteristics of iron and nickel determine the ternary system: iron is in its surface behaviour against sulphur more sensitive compared to nickel.

\section{Conclusion}

The surface tension of the ternary system ironnickel-sulphur and its temperature dependence have been determined using the electromagnetic oscillating droplet technique. Additionally the surface tension data of the boundary system iron-sulphur were also presented and compared with those of other workers. The temperature dependence of the surface tension for the iron and iron-nickel melts containing the surface active element sulphur was found generally to be positive. Because of the similar physical properties of pure liquid iron and nickel the surface active element sulphur mainly determines the course of iso-surface tension lines for the iron-nickel-sulphur system.

\section{Acknowledgement}

The authors are grateful to the Deutsche Forschungsgemeinschaft (DFG) for their financial support of this work.

\section{REFERENCES}

1) M. E. Fraser, W. K. Lu, A. E. Hamielec and R. Murarka: Metall. Trans., 2 (1971), 817. 
2) R. Murarka, W. K. Lu and A. E. Hamielec: Metall. Trans., 2 (1971) 2949

3) R. Murarka, W. K. Lu and A. E. Hamielec: Can. Metall. Quart., 14 (1975), 111.

4) Lord B. Rayleigh: Proc. R. Soc., 29 (1879), 71.

5) H. Soda, A. McLean and W. A. Miller: Trans. JIM, 18 (1977), 445.

6) H. Soda, A. McLean and A. Miller: Metall. Trans. B, 9B (1978), 145.

7) A. Kasama, A. McLean and W. A. Miller: Can. Metall. Quart., 19 (1981), 399

8) A. Kasama, A. McLean, W. A. Miller, Z. Morita and M. J. Word: Can. Metall. Quart., 22 (1983), 9.

9) B. J. Keene, K. C. Mills, J. W. Bryant and E. D. Hondros: Can. Metall. Quart., 21 (1982), 393.

10) B. J. Keene: NPL Report DMA(A) 56, Teddingston, U. K., (1982).

11) B. J. Keene, K. C. Mills and R. F. Brooks: Mater. Sci. Tech., 1 (1985), 568 .

12) J. Schade, A. McLean and W. A. Miller: Proc. 115th Annual Meeting of TMS-AIME, (1986), 233.

13) K. Eckler, I. Egry and D. M. Herlach: Mater. Sci. Eng., AI 33 (1991), 718.

14) B. J. Keene, K. C. Mils, A. Kasama, A. McLean and W. A. Miller: Metall. Trans. B, 17B (1986), 159.

15) L. Nogi, K. Ogino, A. McLean and W. A. Miller: Metall. Trans. $B, 17 B$ (1986), 163.

16) S. Krishnan, G. P. Hansen, R. H. Hauge and J. L. Margrave: Metall. Trans. A, 19A (1988), 1939.

17) K. Nogi, W. B. Chung, A. McLean and W. A. Miller: Mater. Trans. JIM, 32 (1991), 164

18) I. Egry: J. Mater. Sci., 26 (1991), 2997
19) I. Egry: DLR Report IB 333-90/4, Köln, FRG, (1990).

20) I. Egry, G. Lohöfer, P. Neuhaus and S. Sauerland: Mater Sci. Forum, 77 (1991), 257.

21) S. Sauerland, K. Eckler and I. Ergy: J. Mater. Sci. Tech., 11 (1992), 330

22) I. Egry, B. Feuerbacher, G. Lohöfer and P. Neuhaus: Proc. the 7th European Symposium in Material Sciences under Microgravity, ESA SP-295, Oxford, U.K., (1990).

23) H. K. Lee: Dissertation, Technical University Berlin, (1992).

24) H. K. Lee, M. G. Frohberg and J. P. Hajra: Steel Res., (1992), in press.

25) H. K. Lee, M. G. Frohberg and J. P. Hajra: Can. Metall. Quart., (1992), in press.

26) D. L. Cummings, and D. A. Blackburn: J. Fluid Mech., 224 (1991), 395

27) E. Fromm and H. Jehn: Brit. J. Appl. Phys., 16 (1965), 653.

28) G. Lohöfer: SIAM J. Appl. Math., 49 (1989), 567.

29) E. A. Guggenheim: Thermodynamics, North-Holland Physics Publishing, 5th Ed., Amsterdamm, (1967), 216.

30) V. V. Tsarevskii and S. I. Popel: Fiz. Metal. Metalloved., 13 (1962), 451

31) F. A. Halden and W. D. Kingery: J. Phys. Chem., 59 (1955), 557.

32) B. F. Dyson: Trans. TMS-AIME, 227 (1963), 1098.

33) K. M. Gupt, V. I. Yavoishi, A. F. Vishkaryov and S. A. Bliznukov: Trans. Indian Inst. Met., 29 (1976), 286.

34) P. Kozakévitch and G. Urbain: Mem. Scient. Revue Metall., 58 (1961), 517.

35) B. J. Keene: Int. Mat. Rev., 33 (1988), 1, or in Ref. (9).

36) C. H. P. Lupis: Chemical Thermodynamics of Materials, Elsevier Science Publishing Co., Inc., New York, (1983), 405.

37) P. Kozakévitch: Liquids, ed. by T. J. Hughel, Elsevier Publishing Co., Amsterdam, Netherlands, (1965), 243. 